\title{
miR-93 inhibits the invasive potential of triple-negative breast cancer cells in vitro via protein kinase WNK1
}

\author{
SUKANYA SHYAMASUNDAR, JIA PEI LIM and BOON HUAT BAY
}

Department of Anatomy, Yong Loo Lin School of Medicine, National University of Singapore, Singapore 117594, Singapore

Received August 16, 2016; Accepted October 10, 2016

DOI: 10.3892/ijo.2016.3761

\begin{abstract}
Despite advances in treatment, the highly metastatic nature of breast tumors has given rise to the urgent need for development of novel therapeutic and prognostic markers. miR-93 is known to regulate the epithelial to mesenchymal transition process and to influence metastatic spread in breast carcinoma, although the exact mechanism(s)/genes involved remain unknown. In the present study, we examined the role of miR-93 in MDA-MB-231 breast cancer cells. Overexpression of mature miR-93-5p in MDA-MB-231 cells decreased cell migratory capability and invasive potential, as well as increased adhesion. In contrast, inhibition of miR-93 induced the opposite effects. miRNA-mRNA target prediction (TargetScan) identified WNK lysine deficient protein kinase 1 (WNK1), which is known to interact with diverse signaling pathways and regulate cell proliferation, survival, angiogenesis and metastasis, as one of the potential targets of miR-93. Furthermore, we showed by luciferase assay that $W N K 1$ is a putative miR-93 target. siRNA mediated silencing of WNK1 also decreased the invasive ability of the cells, suggesting that the effects of miR-93 may be attributed at least in part to decreased WNK1 expression. Further in vivo studies are required to ascertain the miR-93-WNK1-metastasis cascade, that has potential implications in breast cancer therapy.
\end{abstract}

\section{Introduction}

Breast carcinoma is the predominant cancer in women worldwide (1). Despite advances in treatment, metastasis, tumor recurrence and drug resistance are currently the main challenges in breast cancer management (2), with metastasis occurring in almost $50 \%$ of the patients post-therapy (3).

miRNAs are 18-25 nucleotide non-coding RNAs that control gene expression by degradation of mRNA or inhibiting the translation of transcribed RNA into proteins $(4,5)$.

Correspondence to: Dr Boon Huat Bay, Department of Anatomy, Yong Loo Lin School of Medicine, National University of Singapore, 4 Medical Drive, Blk MD10, Singapore 117594, Singapore

E-mail: boon_huat_bay@nuhs.edu.sg

Key words: miRNA-93, WNK1, migration, invasion, metastasis, breast cancer
Aberrant miRNA expression has been found to underlie several cancers, including breast cancer (reviewed in ref. 6). miRNAs are known to regulate breast cancer progression, by acting as promoters or inhibitors of specific processes, including epithelial-mesenchymal transition (EMT), angiogenesis, stemness of cancer stem cells, invasion, metastasis and chemoresistance $(3,6)$.

miRNA-93 (miR-93), a member of the pro-oncogenic miR-106b-25 cluster (comprising miR-106b, miR-93 and miR-25) is overexpressed in several cancers including breast cancer (7), and belongs to the miR-17 family of miRNAs based on sequence similarity (8). The expression of miR-93 was shown to be significantly increased in triple-negative breast cancer (TNBC) patients when compared to normal tissues or non-TNBC tissues, and associated with lymph node metastasis, TNM grade and Ki-67 staining (9), suggesting that miR-93 controls proliferation and metastasis. Furthermore, the expression of miR-93 was found to be increased in ER- or PR- breast cancer patients when compared to hormonal receptor-positive breast cancer patients (10). However, studies have also revealed that miR-93 has contradictory roles in inhibiting breast cancer metastasis, by regulating the proliferation and differentiation of breast cancer stem cells (11). Nonetheless, the exact mechanism of miR-93 or its gene targets that mediate breast cancer metastasis remain largely unknown.

We examined the role of miR-93 in MDA-MB-231 breast cancer cells, a TNBC cell line. Overexpression of miR-93 decreased cell migration and invasion, while, inhibition of miR-93 elicited the opposite effects in MDA-MB-231 cells. WNK lysine deficient protein kinase 1 (WNK1), one of the targets of miR-93 identified by TargetScan prediction, was verified as a putative target by the luciferase assay. Furthermore, we show that siRNA-mediated silencing of $W N K 1$, resulted in decreased invasive ability of these cells, suggesting that miR-93 mediated changes in cell invasion was possibly via WNK1. Taken together, our results unravel a novel relationship between miR-93, WNK1 and metastasis that have potential implications in breast carcinogenesis.

\section{Materials and methods}

Cell culture. MDA-MB-231 cells (HTB-26) were purchased from the American Type Culture Collection (ATCC; Manassas, VA, USA) and were grown in RPMI medium containing $10 \%$ fetal bovine serum (FBS). 
Table I. The primers used for qPCR.

\begin{tabular}{lll}
\hline Gene & \multicolumn{1}{c}{ Forward primer } & \multicolumn{1}{c}{ Reverse primer } \\
\hline GAPDH & GAAGGTGAAGGTCGGAGTCAACG & TGCCATGGGTGGAATCATATTGG \\
JAK1 & ACGAGTGTCTAGGGATGGCT & CGCATCCTGGTGAGAAGGTT \\
STAT3 & CTGTGGGAAGAATCACGCCT & ACATCCTGAAGGTGCTGCTC \\
EZH1 & TTCCTGCTCCAATGCCTCAG & GTGCTTCCACTACGCAGAGT \\
HMGA2 & CAGGAAGCAGCAGCAAGAAC & AGGCAACATTGACCTGAGCA \\
TGFBR2 & CTCATGGAGTTCAGCGAGCA & GCAGCTCTGTGTTTGGTTG \\
CDH1 & ACAGCACGTACACAGCCCTA & GCAGAAGTGTCCCTGTTCCAG \\
CLDN1 & AAGACGATGAGGTGCAGAAGA & ATTCGTACCTGGCATTGACTG \\
CLDN3 & CAACACCATTATCCGGGACT & CAACACCATTATCCGGGACT \\
\hline
\end{tabular}

Transfection of siRNA. MDA-MB-231 cells were seeded at a density of $2.5 \times 10^{5}$ or $3 \times 10^{4}$ cells/well in 6-well plates or 24 -well plates, respectively. Cells were subsequently transfected with ON-TARGETplus SMARTpool siRNA targeting WNK1 and non-targeting siRNA using DharmaFECT (GE Dharmacon. Pittsburgh, PA, USA). The siRNA complexes were prepared in serum-free RPMI and made up to a final concentration of $20 \mathrm{nM}$. The medium was replaced $24 \mathrm{~h}$ post-transfection, and the transfected cells were cultured for 48 or $72 \mathrm{~h}$ as indicated.

Transfection of miRNA. Cells seeded at the same density as above, were reverse transfected with hsa-miR-93-5p mimic/ negative control (Ambion, Austin, TX, USA) or LNA-hsa-miR93-5p/scramble (Exiqon, Vedbaek, Denmark) and Lipofectamine RNAiMAX (mimics) or Lipofectamine 2000 (inhibitor). The miRNA complexes were prepared in Opti-MEM and made up to a final concentration of $30 \mathrm{nM}$. The medium was replaced with RPMI containing 10\% FBS $6 \mathrm{~h}$ post-transfection, and grown for 48 or $72 \mathrm{~h}$ as indicated.

RNA isolation and cDNA conversion. Total RNA $48 \mathrm{~h}$ post-transfection was isolated using either RNAeasy kit or miRNAeasy kit (which included miRNA) (Qiagen, Hilden, Germany). The isolated RNA was quantified on a spectrophotometer (NanoDrop) and converted into cDNA. Briefly, $1 \mu \mathrm{g}$ of RNA was converted into cDNA using the SuperScript III firststrand synthesis system (Invitrogen, Carlsbad, CA, USA) for gene expression analysis. For analyzing miRNA expression, 20 ng of RNA was converted to cDNA using the Universal cDNA synthesis kit (Exiqon).

$q P C R$. The gene primers used in this study are summarized in Table I. Gene expression levels were quantified by real-time RT-PCR using the Fast SYBR-Green Master Mix (Applied Biosystems, Foster City, CA, USA) in 96-well MicroAmp Fast Optical plates (Applied Biosystems) on a 7900HT Fast real-time PCR system (Applied Biosystems). Relative gene expression was determined by the $2^{-\Delta \Delta \mathrm{Ct}}$ method using $G A P D H$ as the control (12).

miRNA expression was quantified by real-time RT-PCR with miRNA primer hsa-miR-93-5p or control primer U6 (Exiqon) and ExiLENT ${ }^{\mathrm{TM}}$ SYBR-Green Master Mix (Exiqon). The rest of the procedure was as described above.
Growth curve analysis using Alamar blue. Growth of transfected cells was monitored using the Alamar blue assay. Briefly, addition of the Alamar blue reagent (Invitrogen) to culture medium at a ratio of 1:10 was carried out $24 \mathrm{~h}$ posttransfection. Cells were then incubated for $3 \mathrm{~h}$ at $37^{\circ} \mathrm{C}$ and $5 \% \mathrm{CO}_{2}$. Fluorescence intensity was then measured at $570 \mathrm{~nm}$ (excitation) and $585 \mathrm{~nm}$ (emission) wavelengths on a microplate reader (SpectraMax; Molecular Devices, Sunnyvale, CA, USA). Subsequently, cells were replenished with fresh medium and the assay was repeated at 48 and $72 \mathrm{~h}$.

Migration and invasion assays. Cells were harvested $48 \mathrm{~h}$ post-transfection. Cells $\left(2 \times 10^{4}\right)$ were then seeded in $200 \mu \mathrm{l}$ of serum-free RPMI medium into the upper chamber of hydrated polycarbonate membrane insets with $8 \mu \mathrm{m}$ pores (Corning, Corning, NY, USA) for migration assay (18 h) or into hydrated matrigel invasion chambers (BD Biosciences, San Jose, CA, USA) for the invasion assay (20 h). Subsequently, cells were fixed with $100 \%$ methanol followed by staining with crystal violet $(0.5 \% \mathrm{w} / \mathrm{v})$. To determine the number of cells that had migrated or invaded, images from the center and four peripheral fields on the membrane were captured using a Nikon SMZ1500 stereomicroscope at x10 magnification and counted.

Cell adhesion assay. 96-well plates were coated with $20 \mu \mathrm{g} / \mathrm{ml}$ collagen type 1 (Invitrogen) overnight at $4^{\circ} \mathrm{C}$. Subsequently, wells were blocked with $1 \%$ BSA for $1 \mathrm{~h}$ after washing in phosphate buffered saline (PBS). Cells $\left(5 \times 10^{4}\right)$ (48 h posttransfection) were then seeded/well (in duplicates) in $100 \mu \mathrm{l}$ of RPMI with $10 \% \mathrm{FBS}$, and allowed to adhere for $40 \mathrm{~min}$ at $37^{\circ} \mathrm{C}$ with $5 \% \mathrm{CO}_{2}$. Following incubation, non-adherent cells in only one replicate were removed by washing with PBS. MTS reagent was then mixed with serum-free RPMI at a ratio of 1:5, and the wells were replaced with this MTS reagent mixture and incubated for a further $2 \mathrm{~h}$. Following incubation, the OD (absorbance) was read at $590 \mathrm{~nm}$ and the percentage of adherent cells was calculated by the formula: [OD of washed well/OD of non-washed well] x 100.

TargetScan prediction. miR-93 gene targets were predicted by the TargetScan human database (release 7.1) using default parameters (http://www.targetscan.org). 

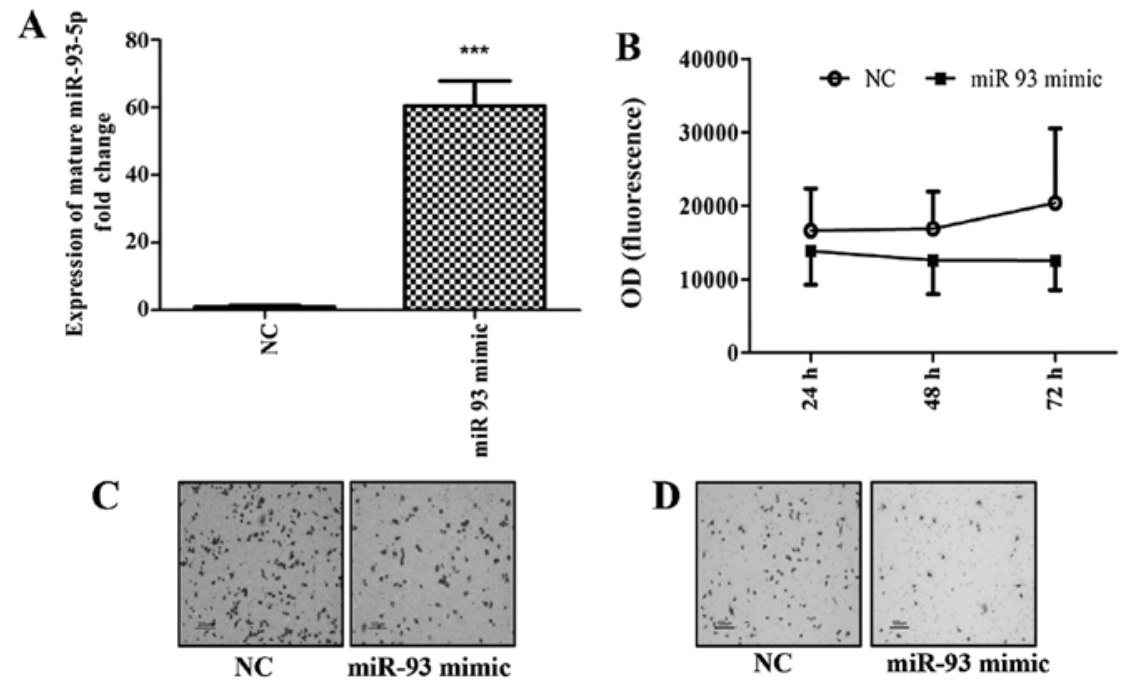

D
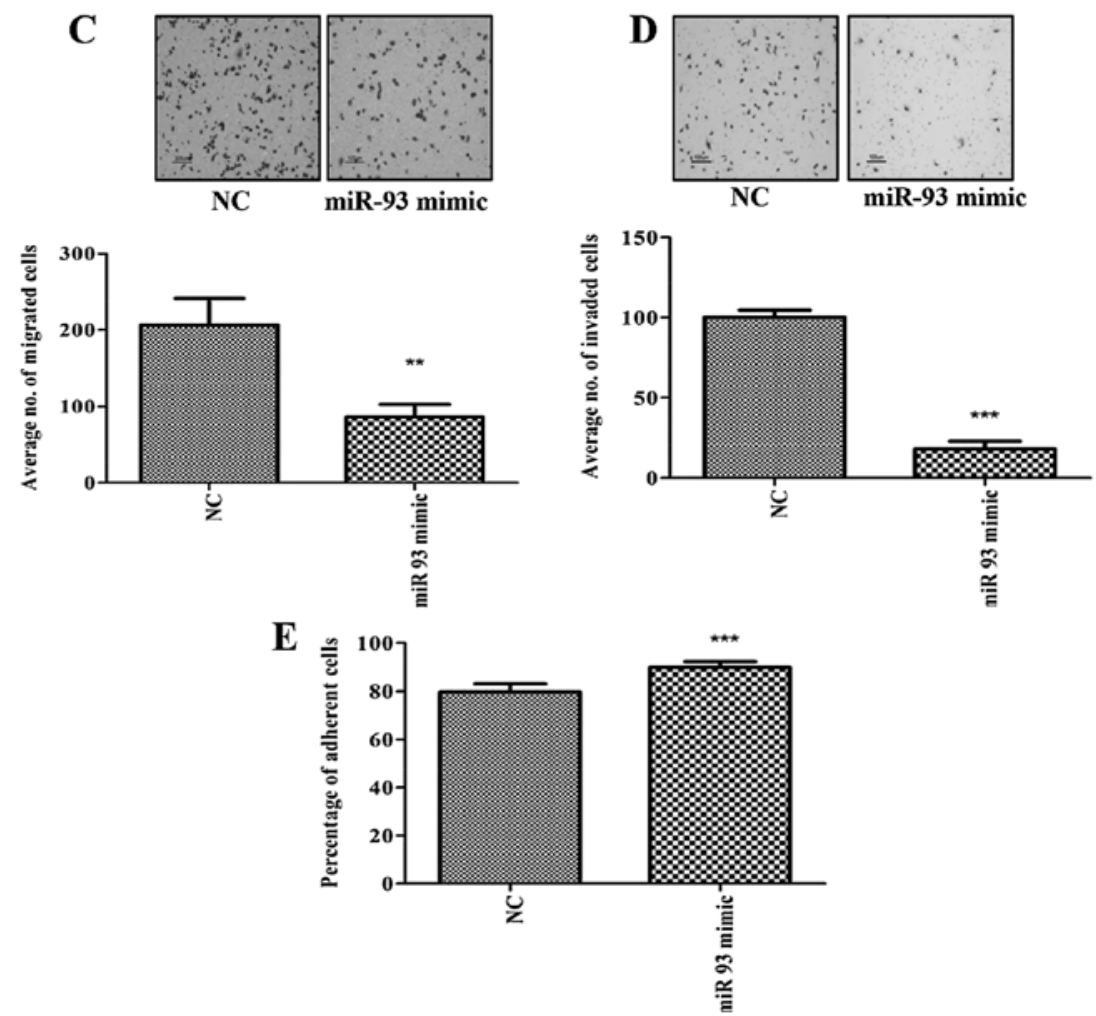

Figure 1. Overexpression of miR-93 decreases migration and invasion, increasing adhesion in MDA-MB-231 breast cancer cells. (A) miR 93 expression was analysed by qPCR, $48 \mathrm{~h}$ post transfection and found to be increased by 60 -fold in miR-93-5p mimic transfected cells compared to negative control (NC) transfected cells. Expression of Sno U6 was used for normalization. (B) Alamar blue assay revealed no significant changes in cell proliferation following miR-93 overexpression. (C and D) Transwell migration or invasion assay was used to assess the ability of cells to migrate (C) and invade (D). Representative images of inserts at x100 magnification are shown on the top. Overexpression of miR-93-5p decreased the migratory ability (C, bottom) and invasive ability of the cells (D, bottom) compared to negative control (NC) mimic transfected cells. (E) miR-93-5p overexpression resulted in increased adhesion of cells to collagen type 1 . Data are presented as mean $\pm \mathrm{SD},{ }^{* *} \mathrm{P}<0.01,{ }^{* * *} \mathrm{P}<0.001$.

3'UTR plasmid luciferase assay. Cells $\left(3 \times 10^{4}\right)$ were plated in 24-well plates and incubated overnight. The next day, $30 \mathrm{nM}$ of negative control mimic or miR-93-5p mimic was co-transfected with $0.3 \mu \mathrm{g}$ of WNK1 plasmid (GeneCopoeia, Rockville, MD, USA) using Lipofectamine 2000. After 24 h, transfected cells were segregated and re-seeded. After another $24 \mathrm{~h}$ post-incubation, the luciferase assay was performed using the Luc-Pair duo luciferase assay kit (GeneCopoeia). The luminescence was read using a spectrophotometer and firefly luciferase was normalized to Renilla luciferase.

Western blot analysis. Protein was extracted at $72 \mathrm{~h}$ post-transfection using the RIPA buffer (Pierce, Waltham, MA, USA) and quantified by the Bradford method (Bio-Rad Laboratories, Hercules, CA, USA). Each sample containing $30 \mu \mathrm{g}$ of protein was denatured at $95^{\circ} \mathrm{C}$ for $5 \mathrm{~min}$ and separated on a 4-20\%
Mini-Protean TGX precast gel (Bio-Rad Laboratories). Proteins were transferred onto a PVDF membrane, which was blocked with 5\% non-fat milk for $1 \mathrm{~h}$ at room temperature and washed well, before incubation with rabbit polyclonal antiWNK1 antibody (1:1000; Abcam, Cambridge, UK) overnight at $4^{\circ} \mathrm{C}$. The next day, secondary anti-rabbit HRP conjugated antibody (Pierce) was added to the blots and incubated for $1 \mathrm{~h}$ at room temperature. Subsequently, development of the blot was carried out using the SuperSignal West Pico chemiluminescence susbtrate (Thermo Fisher Scientific, Waltham, MA, USA) on an automatic film processor. The bands were quantified on a densitometer (Bio-Rad Laboratories) using Quantity One software. To ensure that equal amounts of protein were loaded into each well, the blot was stripped and re-probed with anti- $\beta$-actin antibody (Sigma-Aldrich, St. Louis, MO, USA) to detect $\beta$-actin, the housekeeping protein. 


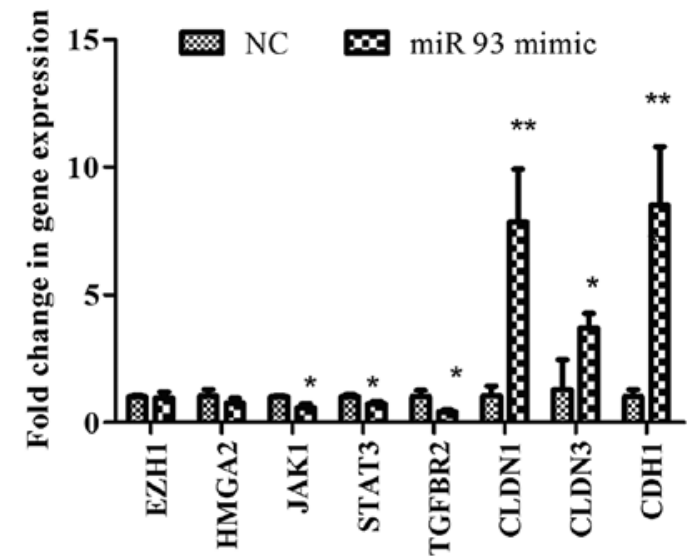

Figure 2. Overexpression of miR-93 altered expression of stem cell and epithelial markers. qPCR analysis revealed decreased expression of $J A K 1$, STAT3 (stem cell markers), and TGFBR2 concomitantly increased expression of $C L D N 1, C L D N 3$ and $C D H 1$ (epithelial markers) following miR-93-5p overexpression in MDA-MB-231 cells. Data are presented as mean $\pm \mathrm{SD}$, ${ }^{*} \mathrm{P}<0.05,{ }^{* *} \mathrm{P}<0.01$.

Statistical analysis. The statistical analysis was performed with GraphPad Prism5 software. Experiments were carried out in triplicates and repeated at least two independent times.
Unpaired, two tailed Student's t-test was used for analysis of data except the growth curves which were analysed by two-way ANOVA. Results are represented as mean $\pm \mathrm{SD}$ and were considered significant at $\mathrm{P}<0.05$.

\section{Results}

Overexpression of miR-93 alters cell migration, invasion and adhesion. Metastasis involves spread of cancer cells from the primary tumor site to distant organs by invading adjacent tissues and extravasating into the circulation (13). We evaluated the effect of miR-93 overexpression on migration, invasion and adhesion, since they are major factors that define the metastatic nature of the cancer cells. We also assessed the cell proliferation as it is a hallmark of cancer.

We first overexpressed miR-93 in MDA-MB-231 using miR-93-5p mimics. The expression of miR-93-5p following transfection was found to be increased by 60 -fold (Fig. 1A). Although overexpression of miR-93-5p in MDA-MB-231 cells did not alter cell proliferation (Fig. 1B), decreased cell migration (Fig. 1C) and cell invasion (Fig. 1D), with increased adhesion of cells to collagen type 1 (Fig. 1E) was observed. In addition, overexpression of miR-93 decreased the expression of stem cell genes, JAK1 and STAT3 and TGFBR2, but
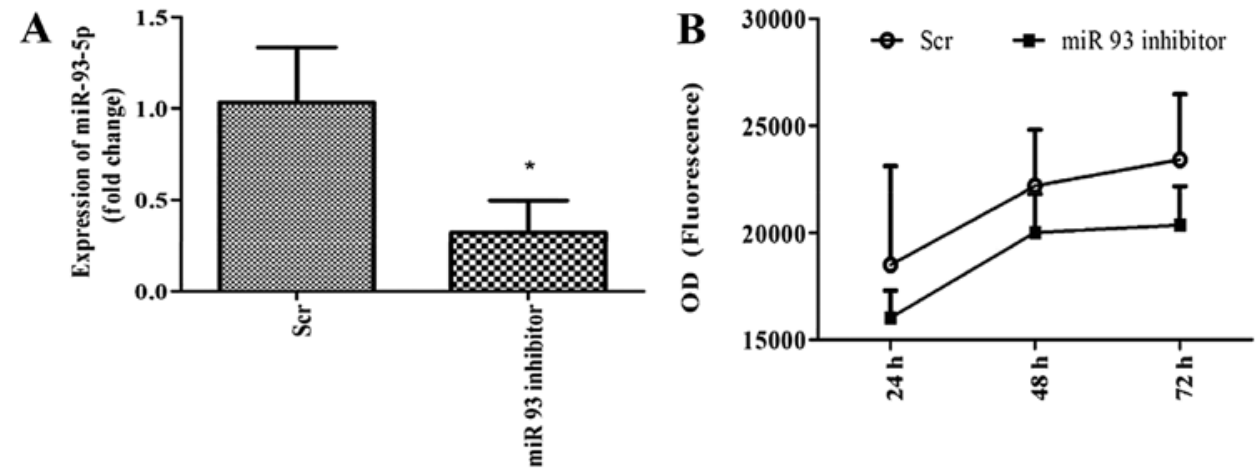
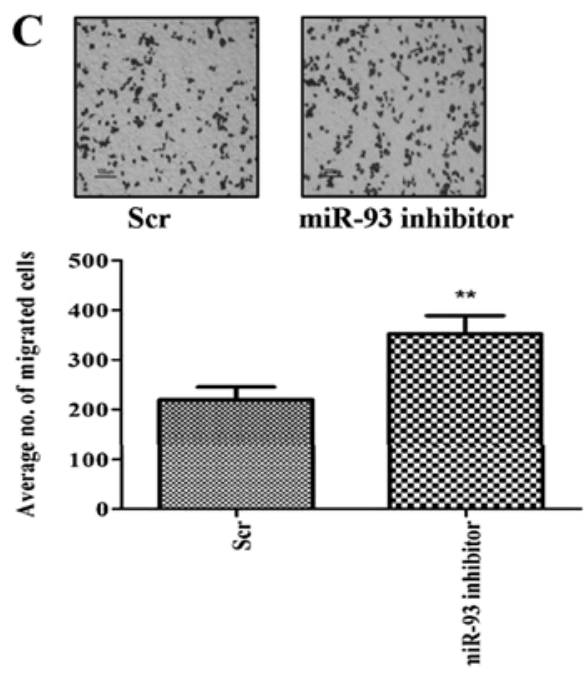

D
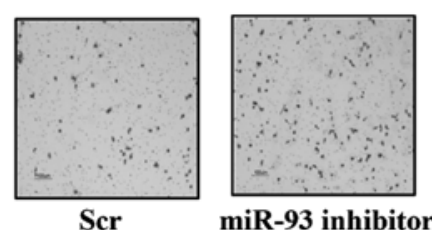

miR-93 inhibitor

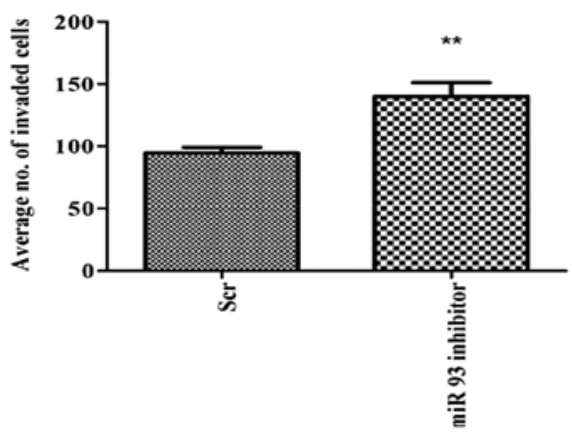

Figure 3. Knockdown of miR-93 increases cell migration and invasion. (A) qPCR analysis revealed that miR-93 expression was decreased by 68\% in miR-93-5p inhibitor transfected cells compared to scrambled control (Scr) transfected cells. U6 expression was used for normalization. (B) Alamar blue assay showed no changes in cell proliferation after miR-93 knockdown as analysed by two-way ANOVA. (C and D) Transwell migration and invasion assay was used to assess the ability of cells to migrate (C) and invade (D). Representative images of inserts at x100 magnification are shown on the top. Knockdown of miR-93-5p increased migration $(C$, bottom panel) and invasion of the cells ( $D$, bottom panel) compared to scrambled control (Scr) transfected cells. Data are presented as mean $\pm \mathrm{SD},{ }^{*} \mathrm{P}<0.05,{ }^{* *} \mathrm{P}<0.01$. 
A
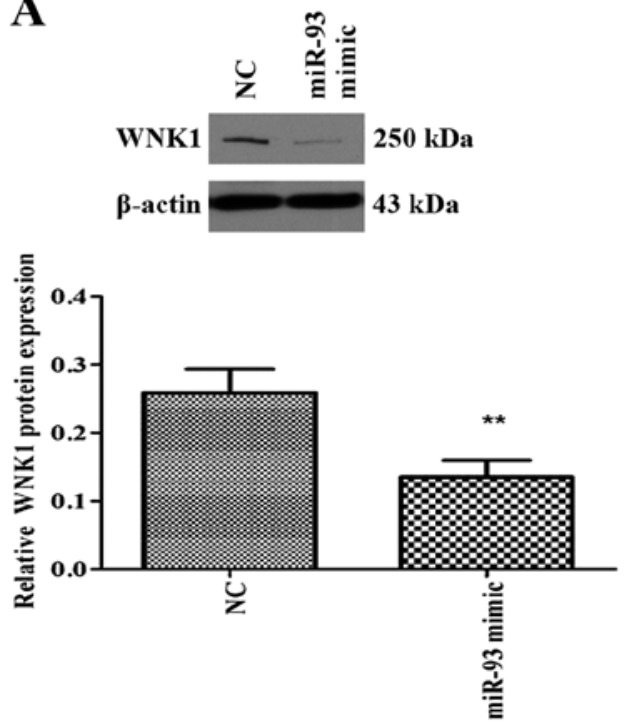

B

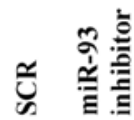
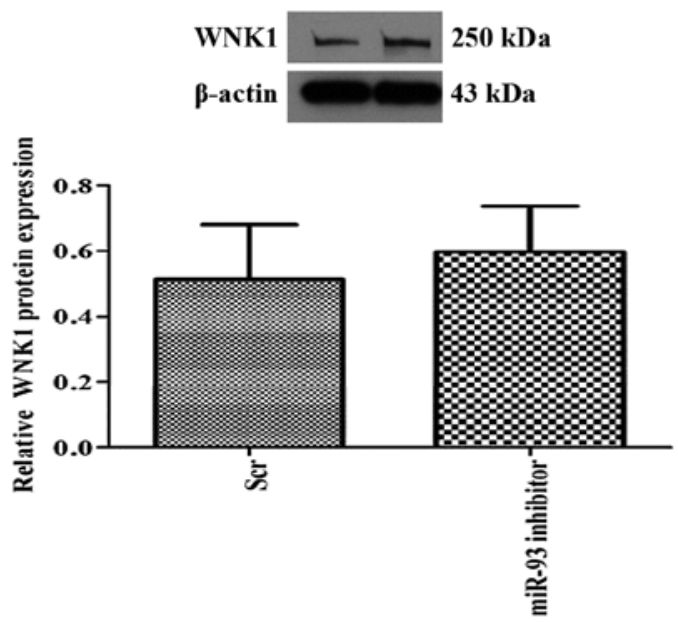

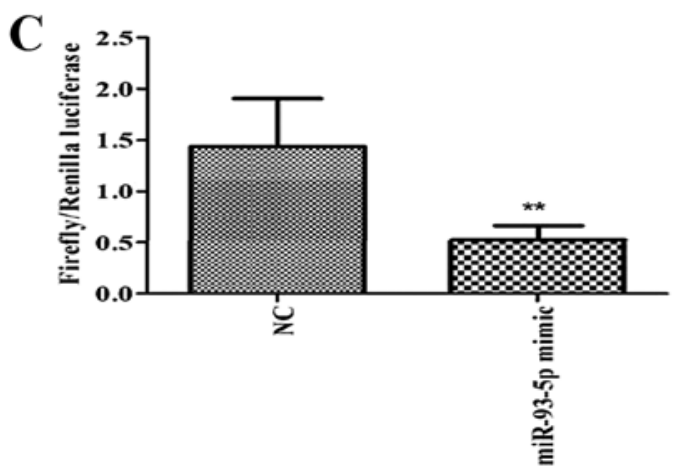

Figure 4. WNK1 is a putative target of miR-93. (A) Overexpression of miR-93-5p decreased WNK1 protein expression. (B) Inhibition of miR-93-5p expression resulted in modest increase in WNK1 protein expression (C) Relative luciferase activity in negative control and miR-93-5p transfected cells. Data are presented as mean $\pm \mathrm{SD},{ }^{* *} \mathrm{P}<0.01$.

increased the $C L D N 1, C L D N 3$ and $C D H 1$ mRNA expression (Fig. 2).

miR-93 knockdown enhances cell migration and invasion. Given that overexpression of miR-93 decreased cell migration and invasion, we inhibited miR-93-5p in MDA-MB-231 cells in order to assess whether this effect could be reversed. Knockdown of miR-93-5p was achieved by transfection of LNA-miR-93-5p inhibitor into the cells and the knockdown efficiency was estimated by qPCR to be $\sim 68 \%$ (Fig. 3A). As expected, inhibition of miR-93 had no effect on cell growth (Fig. 3B), but increased cell migration (Fig. 3C) and invasion (Fig. 3D) compared to scrambled transfected cells (controls).

WNK1 is a target of miR-93-5p. miRNA-mRNA target prediction (TargetScan) revealed that several members of the miR106b 25 and miR-17 family including miR-93, could target two protein kinases, namely $W N K 1$ and $W N K 3$. Since $W N K 1$ is ubiquitously expressed (14) compared to WNK3, we examined the effect of miR-93 on WNK1 expression. Overexpression of miR-93-5p significantly decreased the expression of WNK1 protein (Fig. 4A), suggesting that WNK1 may be a direct or indirect target of miR-93. On the contrary, inhibition of miR-93-5p resulted in a modest increase in WNK1 protein expression (Fig. 4B). In order to determine if
WNK1 is a putative target of miR-93, we performed 3'UTR luciferase assay, which confirmed that miR-93 binds directly to the 3'UTR of WNK1 gene and inhibited its expression (Fig. 4C).

WNK1 knockdown decreased cell migration and invasion. In order to evaluate the effect of WNK1 depletion in MDA-MB-231, we inhibited WNKI expression using siRNA and performed cell migration and invasion assays. Although siRNA-mediated silencing of the WNK1 gene induced no alteration in cell proliferation (Fig. 5A) or migration (Fig. 5B), a significant reduction in the invasive ability of the cells was observed (Fig. 5C).

\section{Discussion}

miRNAs are known to function as tumor suppressors or oncogenes that regulate pathogenesis and progression in several cancers, including breast cancer (15). Thus, miRNAs and/or their targets may serve as novel anticancer targets for therapeutic intervention. In the present study, overexpression of miR-93 was observed to decrease migration, invasion and increase adhesion in parallel, suggesting that miR-93 functions as a tumor suppressor and a negative regulator of metastasis in MDA-MB-231 cells. On the other hand, inhibition of miR-93 
A
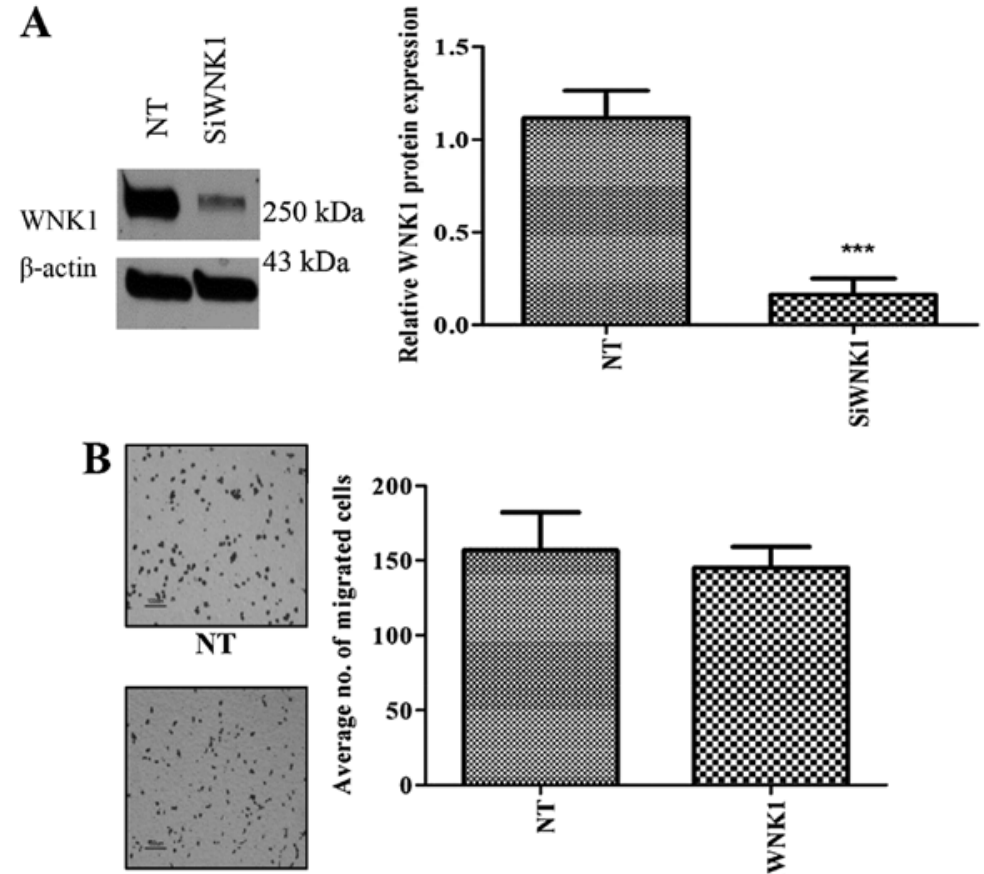

SiWNK1
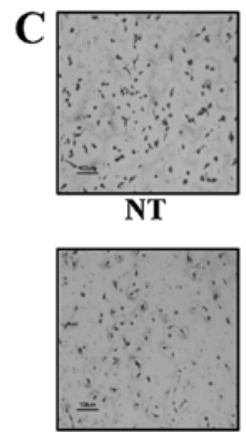

SiWNK1
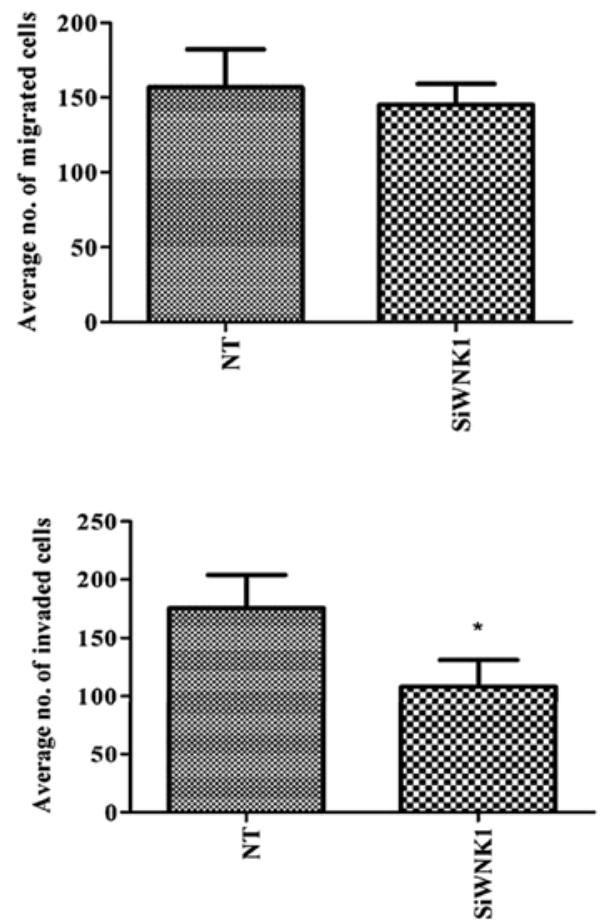

Figure 5. Silencing of the WNK1 gene decreases cell invasion. (A) Representative western blot analysis shows that the expression and quantity of WNK1 protein was decreased following WNK1 silencing. (B and C) Transwell migration and invasion assay was used to assess the ability of cells to migrate (B) and invade (C). Representative images of inserts at x100 magnification are shown on the left. Knockdown of WNK1 did not alter cell migration (B) but decreased cell invasion (C) compared to control cells. Data are presented as mean $\pm \mathrm{SD},{ }^{*} \mathrm{P}<0.05,{ }^{* * *} \mathrm{P}<0.001$.

in MDA-MB-231 cells increased cell migratory capability and invasive potential, further supporting the notion that miR-93 and its gene targets are involved in inhibiting metastasis. Our results are consistent with Liu et al (11), who reported that overexpression of miR-93 inhibited cell invasion while inhibition of miR-93 promoted invasion in SUM159 cells, another claudin low TNBC cell line. In addition, the same authors found that induction of miR-93 in SUM159 cells inhibited metastasis in NOD/SCID mice, while it promoted tumor growth in MCF-7 cells suggesting that the role of miR-93 was both cell line- and differentiation state specific. Nonetheless, the role of miR-93 in MDA-MB-231 breast cancer cells has not been reported previously.

Induction of EMT has been shown to increase characteristics of stem/progenitor cells (16) and high miR-93 (and miR-106b) expression has been found to be associated with stem cell-related genes (17) and EMT-related genes (18) in breast cancer, suggesting that miR-93 and miR-106b regulate these two processes. MDA-MB-231 cells are known to contain a higher percentage of EMT-like CD44+CD24- cancer stem cells that are associated with their increased malignant and metastatic phenotype (19). However, in the present study, miR-93 overexpression in MDA-MB-231 cells was associated with decreased expression of stem cell-related genes (JAKI and STAT3) and TGFBR2 (TGF $\beta$ signaling), and increased expression of epithelial markers ( $C D H 1, C L D N 1$ and $C L D N 3)$, suggesting its involvement in the MET process as reported earlier by Liu et al (11). Furthermore, miR-93 is known to regulate MET during re-programming of fibroblasts to IPS cells via downregulation of its target $T G F B R 2,(20)$ suggesting that miR-93 may be critical for MET in various scenarios. Moreover, miR-93 (and miR-17 family) has been observed to regulate differentiation of stem cells during embryonic development in mice, via downregulating the expression of STAT3 (21), suggesting that miR-93 regulates differentiation of stem cells through STAT3 in different cell types.

In breast cancer, miR-93 has been identified as a basal sub-type specific miRNA by a meta-analysis involving three independent studies (6). However, another recent meta-analysis has also revealed that the miR-17 family of miRNAs that consists 


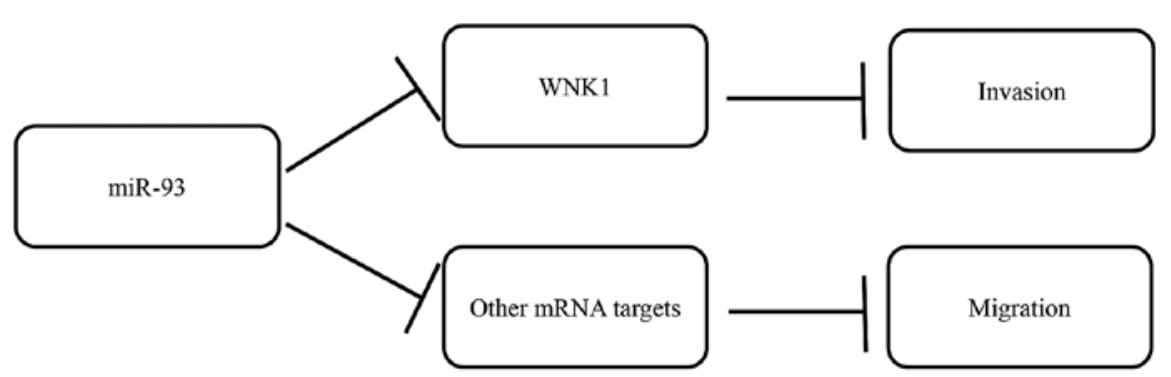

Figure 6. miR-93 regulates cell invasion via inhibiting its target WNK1 and cell migration via other unknown gene targets.

of 6 miRNAs (miR-17-5p, miR-20a, miR-20b, miR-106a, miR$106 \mathrm{~b}$ and miR-93) inhibit metastasis of basal-like tumors by repressing genes involved in EMT (22). The authors showed that overexpression of miR-17-5p suppresses breast cancer metastasis by inhibiting the expression of pro-metastatic genes. In addition, several studies have shown that in breast cancer, miR-17-5p is a tumor suppressor and inhibits proliferation (23), and that miR-17/20 has an anti-invasive role (24). Since miR-93 shares the same seed sequence with miR-17, it is possible that miR-93 inhibits breast cancer metastasis via the same gene targets.

Published literature suggests that miR-93 can act as a tumor suppressor or an oncogene depending on the tumor type, and thus, has contradictory roles in promoting or inhibiting metastasis. In colon cancer, overexpression of miR- 93 has been shown to suppress the proliferation and colony forming ability of colon cancer stem cells (25) and also inhibit growth, migration, invasion and recurrence of colorectal cancer $(26,27)$, while miR-93 promotes proliferation, migration and invasion of nasopharyngeal cancer (28). Nevertheless, what remains consistent are that the gene targets of the miRNAs from the miR-106b 25 cluster and miR-17 family across several cancers (for example, E2F1, CCNB1, p21, BIM, TGFBR2 are regulated by miR-93 in breast, colorectal, gastric and nasopharyngeal cancers among others) (26,29-31), suggesting that the miRNA expression is differentially regulated (inhibited or overexpressed) in cancer, resulting in upregulation or downregulation of its targets (that are oncogenes or tumor suppressors respectively), which are critical for cancer progression.

Humans contain four different $W N K$ genes (WNK1-4), among which only $W N K l$ is ubiquitously expressed in all tissues (14). Loss of WNK1 during development has been shown to result in embryonic lethality in mice (32) and zebrafish embryos (33), suggesting that WNK1 is critical for development. Among the WNKs, WNK1 is known to interact with diverse signaling pathways such as Smad/Tgfb (34), Erk5/MAPK (35) and PI-3K pathways (36) to regulate cell proliferation, survival, angiogenesis and metastasis, suggesting that WNK1 has important roles in tumorigenesis. Studies have shown that WNK1, is an important protein kinase that is required for mitosis and abscission (37), migration and invasion of neural tumor cells via ganglioside GD3 (38), migration of glioma cells (39) and migration, angiogenesis and EMT in endothelial cells (40). Furthermore, WNK1 has been shown to regulate Slug, Zeb1 and $\beta$-catenin in endothelial cells (40), with the expression levels of Slug increasing in the presence of WNK1. Slug has well-known roles in tumor invasion (41) and thus, provides an important link between WNK1 and metastasis.
In addition, silencing of WNK1 in a mouse progenitor cell line inhibited differentiation of the cells into neuronal and glial lineage, and upregulated the expression of stem cells/ progenitor marker nestin (42). Recently, inhibition of ERK5 or inhibition of genes that phosphorylate and activate ERK5, such as MAP3K2 and WNK1 has been shown to reduce tumor growth and metastasis in prostate cancer in vivo (43). Furthermore, depletion of AKT/WNK1 has been shown to revert EMT and inhibit cell migration in lung cancer cells (44). Together, these studies also highlight the importance of WNK1 in the regulation of differentiation, tumor growth and metastasis.

While miR-93 has several gene targets, we postulate that the effects on migration and invasion following miR-93 overexpression were possibly mediated via decreasing WNK1 (identified from target prediction). Hence, we inhibited WNK1 expression using siRNA in the MDA-MB-231 cells and observed decreased invasive ability, but not cell migration, suggesting that the effects of miR-93 overexpression in reducing cell invasion was mediated via decreasing the expression of WNK1 post-transcriptionally (Fig. 6). It would appear that decrease in migration observed after miR-93 overexpression was not brought about by WNK1, but possibly through other miR-93 gene targets that remain to be elucidated.

In summary, we have demonstrated that overexpression of miR-93 decrease cell migration and invasion in MDA-MB-231 breast cancer cells in vitro. We have identified WNK1 as a novel target of miR-93 that mediates cell invasion. Further in vivo studies are required to ascertain the miR-93-WNK1metastasis cascade that has potential implications in breast cancer therapy.

\section{Acknowledgements}

The present study was supported by the Ministry of Education grant (MOE2013-T2-1-129). Jia Pei Lim is a recipient of the Ong Hin Tiang Scholarship in Cancer Research.

\section{References}

1. Bombonati A and Sgroi DC: The molecular pathology of breast cancer progression. J Pathol 223: 307-317, 2011.

2. André $F$ and Zielinski CC: Optimal strategies for the treatment of metastatic triple-negative breast cancer with currently approved agents. Ann Oncol 23 (Suppl 6): vi46-vi51, 2012.

3. Lin Y, Liu AY, Fan C, Zheng H, Li Y, Zhang C, Wu S, Yu D, Huang Z, Liu F, et al: MicroRNA-33b inhibits breast cancer metastasis by targeting HMGA2, SALL4 and Twist1. Sci Rep 5: 9995, 2015. 
4. Bartel DP: MicroRNAs: Target recognition and regulatory functions. Cell 136: 215-233, 2009.

5. Zhong X, Coukos G and Zhang L: miRNAs in human cancer. Methods Mol Biol 822: 295-306, 2012.

6. van Schooneveld E, Wildiers H, Vergote I, Vermeulen PB, Dirix LY and Van Laere SJ: Dysregulation of microRNAs in breast cancer and their potential role as prognostic and predictive biomarkers in patient management. Breast Cancer Res 17: 21, 2015.

7. Fang L, Du WW, Yang W, Rutnam ZJ, Peng C, Li H, O'Malley YQ, Askeland RW, Sugg S, Liu M, et al: MiR-93 enhances angiogenesis and metastasis by targeting LATS2. Cell Cycle 11: 4352-4365, 2012.

8. Smith AL, Iwanaga R, Drasin DJ, Micalizzi DS, Vartuli RL, Tan AC and Ford HL: The miR-106b-25 cluster targets Smad7, activates TGF- $\beta$ signaling, and induces EMT and tumor initiating cell characteristics downstream of Six1 in human breast cancer. Oncogene 31: 5162-5171, 2012.

9. Hu J, Xu J, Wu Y, Chen Q, Zheng W, Lu X, Zhou C and Jiao D: Identification of microRNA-93 as a functional dysregulated miRNA in triple-negative breast cancer. Tumour Biol 36: 251-258, 2015

10. Kolacinska A,Morawiec J,Pawlowska Z,SzemrajJ,Szymanska B, Malachowska B, Morawiec Z, Morawiec-Sztandera A, Pakula L, Kubiak R, et al: Association of microRNA-93, 190, 200b and receptor status in core biopsies from stage III breast cancer patients. DNA Cell Biol 33: 624-629, 2014.

11. Liu S, Patel SH, Ginestier C, Ibarra I, Martin-Trevino R, Bai S, McDermott SP, Shang L, Ke J, Ou SJ, et al: MicroRNA93 regulates proliferation and differentiation of normal and malignant breast stem cells. PLoS Genet 8: e1002751, 2012.

12. Livak KJ and Schmittgen TD: Analysis of relative gene expression data using real-time quantitative PCR and the 2(-Delta Delta C(T)) method. Methods 25: 402-408, 2001.

13. Kang Y and Massagué J: Epithelial-mesenchymal transitions: Twist in development and metastasis. Cell 118: 277-279, 2004.

14. Moniz S and Jordan P: Emerging roles for WNK kinases in cancer. Cell Mol Life Sci 67: 1265-1276, 2010.

15. Wang W and Luo YP: MicroRNAs in breast cancer: Oncogene and tumor suppressors with clinical potential. J Zhejiang Univ Sci B 16: 18-31, 2015.

16. Mani SA, Guo W, Liao MJ, Eaton EN, Ayyanan A, Zhou AY, Brooks M, Reinhard F, Zhang CC, Shipitsin M, et al: The epithelial-mesenchymal transition generates cells with properties of stem cells. Cell 133: 704-715, 2008

17. Wong DJ, Liu H, Ridky TW, Cassarino D, Segal E and Chang HY Module map of stem cell genes guides creation of epithelial cancer stem cells. Cell Stem Cell 2: 333-344, 2008.

18. Sarrió D, Rodriguez-Pinilla SM, Hardisson D, Cano A, MorenoBueno G and Palacios J: Epithelial-mesenchymal transition in breast cancer relates to the basal-like phenotype. Cancer Res 68: 989-997, 2008

19. Kuperwasser C, Dessain S, Bierbaum BE, Garnet D, Sperandio K, Gauvin GP, Naber SP, Weinberg RA and Rosenblatt M: A mouse model of human breast cancer metastasis to human bone. Cancer Res 65: 6130-6138, 2005

20. LiZ, Yang CS, Nakashima K and Rana TM: Small RNA-mediated regulation of iPS cell generation. EMBO J 30: 823-834, 2011.

21. Foshay KM and Gallicano GI: miR-17 family miRNAs are expressed during early mammalian development and regulate stem cell differentiation. Dev Biol 326: 431-443, 2009.

22. Fan M, Sethuraman A, Brown M, Sun W and Pfeffer LM: Systematic analysis of metastasis-associated genes identifies miR-17-5p as a metastatic suppressor of basal-like breast cancer. Breast Cancer Res Treat 146: 487-502, 2014.

23. Hossain A, Kuo MT and Saunders GF: Mir-17-5p regulates breast cancer cell proliferation by inhibiting translation of AIB1 mRNA. Mol Cell Biol 26: 8191-8201, 2006.

24. Yu Z, Willmarth NE, Zhou J, Katiyar S, Wang M, Liu Y, McCue PA, Quong AA, Lisanti MP and Pestell RG: microRNA $17 / 20$ inhibits cellular invasion and tumor metastasis in breast cancer by heterotypic signaling. Proc Natl Acad Sci USA 107: $8231-8236,2010$

25. Yu XF, Zou J, Bao ZJ and Dong J: miR-93 suppresses proliferation and colony formation of human colon cancer stem cells World J Gastroenterol 17: 4711-4717, 2011.
26. Yang IP, Tsai HL, Hou MF, Chen KC, Tsai PC, Huang SW, Chou WW, Wang JY and Juo SH: MicroRNA-93 inhibits tumor growth and early relapse of human colorectal cancer by affecting genes involved in the cell cycle. Carcinogenesis 33: 1522-1530, 2012.

27. Tang Q, Zou Z, Zou C, Zhang Q, Huang R, Guan X, Li Q, Han Z, Wang D, Wei H, et al: MicroRNA-93 suppress colorectal cancer development via Wnt/ $\beta$-catenin pathway downregulating. Tumour Biol 36: 1701-1710, 2015.

28. Xu YF, Mao YP, Li YQ, Ren XY, He QM, Tang XR, Sun Y, Liu N and Ma J: MicroRNA-93 promotes cell growth and invasion in nasopharyngeal carcinoma by targeting disabled homolog-2. Cancer Lett 363: 146-155, 2015.

29. Zhang $\mathrm{H}$ and Yan X: Cantharidin modulates the E2F1/MCM7miR-106b-93/p21-PTEN signaling axis in MCF-7 breast cancer cells. Oncol Lett 10: 2849-2855, 2015.

30. Petrocca F, Vecchione A and Croce CM: Emerging role of miR-106b-25/miR-17-92 clusters in the control of transforming growth factor beta signaling. Cancer Res 68: 8191-8194, 2008.

31. Lyu X, Fang W, Cai L, Zheng H, Ye Y, Zhang L, Li J, Peng H, Cho WC, Wang E, et al: TGF $\beta$ R2 is a major target of miR-93 in nasopharyngeal carcinoma aggressiveness. Mol Cancer 13: 51, 2014.

32. Xie J, Wu T, Xu K, Huang IK, Cleaver O and Huang CL: Endothelial-specific expression of WNK1 kinase is essential for angiogenesis and heart development in mice. Am J Pathol 175: 1315-1327, 2009.

33. Lai JG, Tsai SM, Tu HC, Chen WC, Kou FJ, Lu JW, Wang HD, Huang CL and Yuh CH: Zebrafish WNK lysine deficient protein kinase 1 (wnk1) affects angiogenesis associated with VEGF signaling. PLoS One 9: e106129, 2014.

34. Lee BH, Chen W, Stippec S and Cobb MH: Biological cross-talk between WNK1 and the transforming growth factor beta-Smad signaling pathway. J Biol Chem 282: 17985-17996, 2007.

35. Wilson FH, Disse-Nicodème $\mathrm{S}$, Choate KA, Ishikawa K, NelsonWilliams C, Desitter I, Gunel M, Milford DV, Lipkin GW, Achard JM, et al: Human hypertension caused by mutations in WNK kinases. Science 293: 1107-1112, 2001.

36. Xu BE, Stippec S, Chu PY, Lazrak A, Li XJ, Lee BH, English JM, Ortega B, Huang CL and Cobb MH: WNK1 activates SGK1 to regulate the epithelial sodium channel. Proc Natl Acad Sci USA 102: 10315-10320, 2005.

37. Tu SW, Bugde A, Luby-Phelps K and Cobb MH: WNK1 is required for mitosis and abscission. Proc Natl Acad Sci USA 108: 1385-1390, 2011

38. Zeng G, Gao L and Yu RK: Reduced cell migration, tumor growth and experimental metastasis of rat F-11 cells whose expression of GD3-synthase is suppressed. Int J Cancer 88: 53-57, 2000.

39. Zhu W, Begum G, Pointer K, Clark PA, Yang SS, Lin SH, Kahle KT, Kuo JS and Sun D: WNK1-OSR1 kinase-mediated phospho-activation of $\mathrm{Na}^{+}-\mathrm{K}^{+}-2 \mathrm{Cl}^{-}$cotransporter facilitates glioma migration. Mol Cancer 13: 31, 2014.

40. Dbouk HA, Weil LM, Perera GK, Dellinger MT, Pearson G, Brekken RA and Cobb MH: Actions of the protein kinase WNK1 on endothelial cells are differentially mediated by its substrate kinases OSR1 and SPAK. Proc Natl Acad Sci USA 111: 15999-16004, 2014.

41. Alves CC, Carneiro F, Hoefler H and Becker KF: Role of the epithelial-mesenchymal transition regulator Slug in primary human cancers. Front Biosci (Landmark Ed) 14: 3035-3050, 2009.

42. Sun X, Gao L, Yu RK and Zeng G: Down-regulation of WNK1 protein kinase in neural progenitor cells suppresses cell proliferation and migration. J Neurochem 99: 1114-1121, 2006

43. Fulford L, Milewski D, Ustiyan V, Ravishankar N, Cai Y, Le T, Masineni S, Kasper S, Aronow B, Kalinichenko VV, et al: The transcription factor FOXF1 promotes prostate cancer by stimulating the mitogen-activated protein kinase ERK5. Sci Signal 9: ra48, 2016.

44. Hsu YL, Hung JY, Chiang SY, Jian SF, Wu CY, Lin YS, Tsai YM, Chou SH, Tsai MJ and Kuo PL: Lung cancer-derived galectin-1 contributes to cancer associated fibroblast-mediated cancer progression and immune suppression through TDO2/kynurenine axis. Oncotarget 7: 27584-27598, 2016. 